2012 were included in the audit. Data was collected via the RRT database and Retrieval referral forms.

Results Over twenty months NWTS received 1637 referrals from 29 District General Hospitals in the region out of which 20 patients $(1.22 \%)$ were severe DKA. 6/20 were newly diagnosed DKA.

At time of referral 10/20 were noted to have significantly reduced GCS, requiring hypertonic saline and an urgent CT scan.

Following advice from NWTS, only 7 were transferred to PICU and rest were managed in their local DGH; each followed up for a period of $24-48$ hours with minimum of two follow-up calls.

3 out of 7 transferred to PICU had very low level of consciousness (GCS 3-4/15); the rest had intercurrent illness e.g. sepsis, myocarditis and non availability of HDU beds.

All patients survived and only one patient (who also had problems with severe tachyarrhythmia and associated poor cardiac output) had evidence of neurological injury.

Conclusion With advice and support the local DGH team can be guided through the management of severe DKA including those with reduced GCS, with good outcome.

The DGH clinicians value the support available from Regional Retrieval Team as many are unfamiliar with management of such rare situations.

The majority of patients did not require transfer to tertiary centres which benefited both the patient and family and allows best utilisation of resources.

\section{G62(P) RETROSPECTIVE ANALYSIS OF PICU READMISSIONS OVER A ONE YEAR PERIOD}

doi:10.1136/archdischild-2013-304107.074

CJ Trivers, J Owen, R Levin. Paediatric Intensive Care Unit, Roval Hospital for Sick Children, Glasgow, UK

Aims Readmission may suggest premature discharge and may be detrimental to the patient's care. Our aim was to determine our PICU readmission rate and review the patterns of readmission.

Methods A retrospective case series analysis was performed. Patients with multiple admissions during September 2010 to August 2011 were identified using the computerised information system, CIS.
Patients who had been discharged home or had an elective readmission were excluded. The CIS and hospital case notes of the patients were analysed using standard proformas.

Information recorded included readmission reason, length of stay, time from discharge to readmission, CEWS scores prior to discharge from PICU and intervention required in PICU (figures 1, 2, 3).

Results 39 patients were readmitted which represents a readmission rate of $3.8 \% .10$ patients were readmitted within 24 hours of discharge (26\%),14 were readmitted within 3 days (36\%) and the remainder between 4 and 7 days. Average time of readmission was $60 \mathrm{hrs}$ (mean) with a range of 3 hrs to 168 hours.

The majority $(23-60 \%)$ of the patients were readmitted with respiratory distress. 3 patients were readmitted with cardiac failure. 4 patients were readmitted with life threatening events (10\%)

The majority of patients were cardiac ( $52 \%)$.

The majority of patients had a CEW score of 0 prior to discharge.

Almost half of the patients $(46 \%)$ readmitted required ventilation,12 required inotropic support. The rest required observation only.

9 patients were readmitted for less than 24 hours (23\%), 10 (25\%) patients for between 1 and 3 days and 20 (51\%) for more than 3 days. The longest readmission was 73 days, median 202 hours.

Conclusion A readmission rate of $3.8 \%$ is within the accepted norm although there is a lack of paediatric data.

Most of our patients were readmitted with respiratory distress usually caused by the same condition necessitating initial admission. The majority of our readmissions occurred within 72 hours. The patients that did not require ventilation had a generally short admission (mean $38 \mathrm{hrs}$ ). There was a large group,however, who required a lot of intervention and had a prolonged second admission. We did not identify any clear avoidable risk factors for readmission.

\section{G63(P) INVESTIGATION OF FAMILIAL VESICO-URETERIC REFLUX IN INFANTS, IS IT WORTHWHILE?}

doi:10.1136/archdischild-2013-304107.075

'R Hubbard, 'N Collins, 'D Matthew, 'L Baines, 'H Lambert. 'The Great North Childrens' Hospital, Newcastle Hospitals NHS Trust, Newcastle upon Tyne, UK; '2Department of Nephrology, Newcastle Hospitals NHS Trust, Newcastle upon Tyne, UK

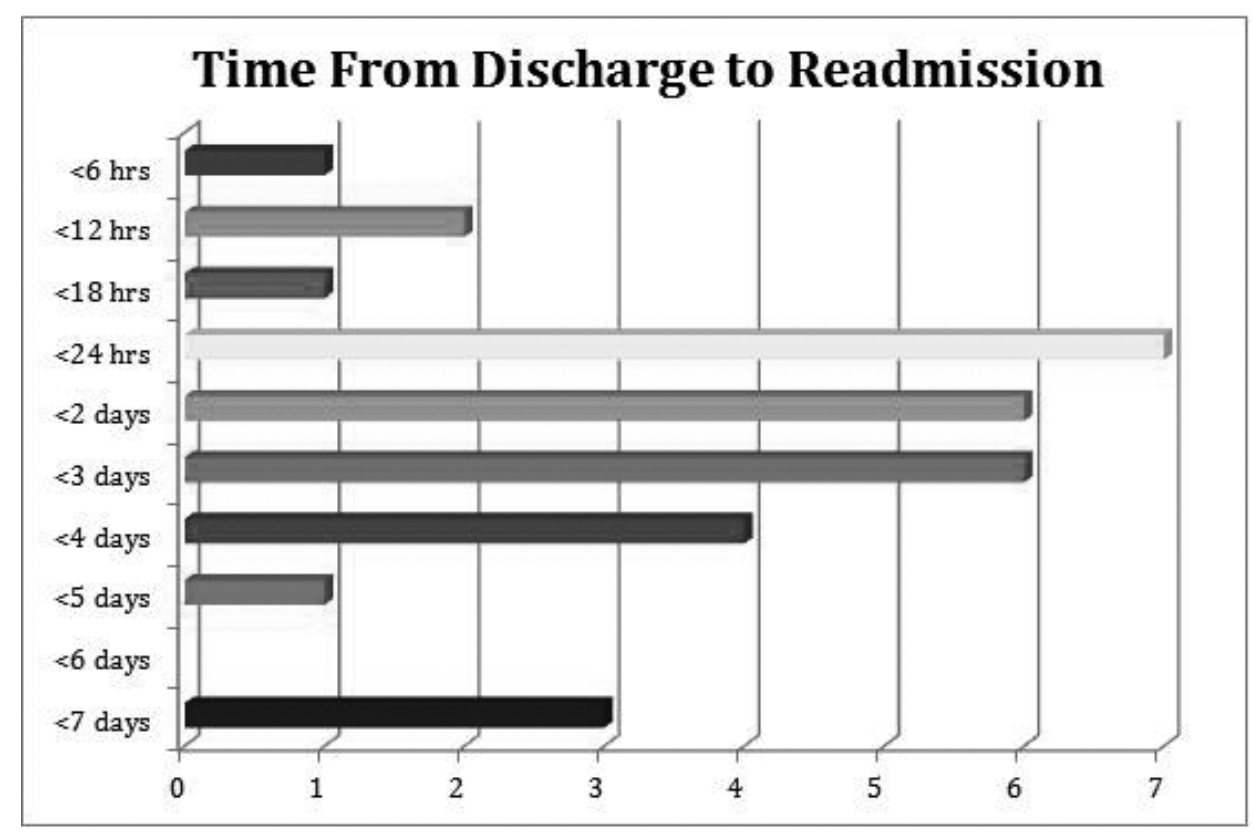

